\title{
An updated and annotated list of Indian lizards (Reptilia: Sauria) based on a review of distribution records and checklists of Indian reptiles
}

\section{P. Dilip Venugopal} Department of Entomology, University of Maryland, 4124 Plant Sciences Building, College Park, MD 20742-4454, USA
Email: dilip@umd.edu

Date of publication (online): 26 March 2010 Date of publication (print): 26 March 2010 ISSN 0974-7907 (online) | 0974-7893 (print)

Editor: Aaron Bauer

\section{Manuscript details:}

Ms \# 02083

Received 21 October 2008

Final received 31 December 2009

Finally accepted 14 February 2010

Citation: Venugopal, P.D. (2010). An updated and annotated list of Indian lizards (Reptilia: Souria) based on a review of distribution records and checklists of Indian reptiles. Journal of Threatened Taxa 2(3): 725-738.

Copyright: ( ) P. Dilip Venugopal 2010. Creative Commons Attribution 3.0 Unported License. JoTT allows unrestricted use of this article in any medium for non-profit purposes, reproduction and distribution by providing adequate credit to the authors and the source of publication.

Author Details: P. DILIP VENUGOPAL is a graduate student at the Department of Entomology, University of Maryland, College Park. His broad interests include understanding spatial patterns in species distributions, as influenced by trophic interactions and environmental variables.

Acknowledgements: I thank Dr. N.M. Ishwar Narayan for initiating me into this work and for providing literature and valuable inputs over the past few years. I thank Dr. Indraneil Das and Dr. Peter Uetz for clearing doubts on taxonomic status and distribution of many species and also for providing literature. Discussions with Dr. B. R. Ramesh, Dr. Ranjit Daniels, Shreyas Krishnan, Robin Vijayan and Sara Lombardi helped improving this manuscript. The library staff including the librarian of the Wildlife Institute of India provided access and assistance. I thank Harry Andrews and the staff of Madras Crocodile Bank Trust for access to the library and logistic support; Kalaiarasan for providing access to the Chennai Snake Part Trust library. I thank the members of the HISASIA web discussion forum for help in clarifications and literature collection V. Pattabhiraman provided logistical support at different stages of this work. Many individuals helped in literature collection - Karthik Ram, Vinatha Viswanathan, Shreyas Krishnan, Bindu Raghavan, S. U. Saravanakumar, Nandin Rajamani, Robin Vijayan and several individual authors who sent me their publications. Reviewers comments helped improve the manuscript

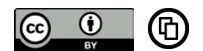

OPEN ACCESS | FREE DOWNLOAD
Abstract: Over the past two decades many checklists of reptiles of India and adjacent countries have been published. These publications have furthered the growth of knowledge on systematics, distribution and biogeography of Indian reptiles, and the field of herpetology in India in general. However, the reporting format of most such checklists of Indian reptiles does not provide a basis for direct verification of the information presented. As a result, mistakes in the inclusion and omission of species have been perpetuated and the exact number of reptile species reported from India still remains unclear. A verification of the current listings based on distributional records and review of published checklists revealed that 199 species of lizards (Reptilia: Sauria) are currently validly reported on the basis of distributional records within the boundaries of India. Seventeen other lizard species have erroneously been included in earlier checklists of Indian reptiles. Omissions of species by these checklists have been even more numerous than erroneous inclusions. In this paper, I present a plea to report species lists as annotated checklists which corroborate the inclusion and omission of species by providing valid source references or notes.

Keywords: Checklists, distributional records, India, lizards, Reptiles, review

\section{INTRODUCTION}

Plant and animal species survey and observational data are vast resources that provide present day and historical information on geographic distribution. Primary species-occurrence data have wide and varied uses, encompassing virtually every aspect of human life - food, shelter and recreation, art and history, society, science and politics (Chapman 2005a). Species listings or checklists, which contain such primary and compiled species-occurrence data, play a vital role in providing information on the number of species occurring in different regions across different spatial scales (local, regional, national and global). Such species occurrence data, in the form of checklists, have been used for taxonomic and biogeographic studies for hundreds of years (Chapman 2005a). Some of the other uses include conservation planning, reserve selection, climate change studies, agriculture, forestry and fishery, and species translocation studies, to name a few (See Chapman 2005a for a detailed account of the uses of species-occurrence data). Accuracy and precision (sensu Chapman 2005b) of the taxonomic and nomenclatural information and the spatial information are important considerations for determination of data quality and validation of the species occurrence data (Chapman 2005b). In this context, the importance of the data quality in the checklist of Indian reptiles - the storehouses of information on the reptilian species occurrence data- hardly needs emphasis.

Among the publications pertaining to reptilian taxonomy and species occurrences in India the works of Malcolm Smith (1931, 1935a, 1943), though more than half a century old, still remains the most important contribution (Das 2003). Over the past two decades many checklists of reptiles of India (Murthy 1985; Murthy 1990; Tikader \& Sharma 1992; Das 1997a; Das 2003), sometimes including adjacent countries (Das 1994; Das1996a; Sharma 2002) 
have been published. These publications have furthered the growth of knowledge on systematics, distribution and biogeography of Indian reptiles, and the field of herpetology in India in general. The primary objective of these publications, except those providing information of species distribution (for example - Murthy 1985; Tikader \& Sharma 1992) and taxonomic development in India (Das 2003), included enlisting the reptilian species occurring in India. However, some of these publications have come under severe criticism (see review by Das 1996b; Pawar 1998), with reservation over the quality of the information presented. Especially the publications of the Zoological Survey of India, which still are followed by many workers in India, have especially been criticized for their failure to follow the changes in the taxonomic and distributional information of species.

An apparent inadequacy of the above-mentioned checklists of Indian reptiles published over the past 20 years is that species with valid distributional records are not differentiated from those with questionable records. While a reference to the taxonomic treatise has been provided (e.g. Das 2003), a valid reference or source for the distribution records corroborating the inclusion and omission of species have not been cited by any of these checklists. It is observed that despite being compilations, neither all of the bibliographic sources referred nor the details on locality records have been provided in most of the checklists (regional, state-wise or national) of Indian reptiles. For example, Vyas (2000) has noted and criticized the absence of source literature within the checklist of Gujarat reptiles provided by Gayen (1999). The failure to acknowledge all the literary sources used for the compilation of the species list is a reproachable attribute of these publications that is tantamount to plagiarism.

In addition to this significant limitation, the distribution records of species pre and post partition of British India have not been distinguished, resulting in erroneous inclusion of many species into the checklist. Incorrect inclusion of species that were not recorded within India, doubtful records and omissions of valid species has also been common. The inclusion or omission of species has not been corroborated with references or notes thereby rendering the information presented unverifiable. As a result, mistakes in species inclusion or omission have been perpetuated, and without any grounds for further verification. I quote Bobrov (2005) to illustrate this point with an example - "Phrynocephalus reticulatus was reported in Ladakh (Smith 1935). Later this single and clearly erroneous finding was mentioned in every publication on the herpetofauna of India, Kashmir and Ladakh." If the publications listing them had provided the source literature, it would have provided an opportunity for verification.

Furthermore, the lack of rationale for inclusion and omission of species has resulted in inconsistencies in the lists contributed by the same individual workers. For example Murthy (1985) erroneously included Dasia grisea in the list of Indian reptiles, with Andaman \& Nicobar Islands as its distributional range. However, the list of reptiles provided by Murthy (1990) rightfully did not include this species. However, it erroneously appeared again in the list provided by Murthy (1994). Similarly Das (1994) \& Das (1996a) did not include India in the distributional range of Phyrnocephalus luteoguttatus, but it was erroneously listed for India by Das (1997a). However this species was not included in a later list by Das (2003).

A direct consequence of the poor reporting standards of the checklists of Indian reptiles is that the exact number of reptilian species with valid distributional records within India still remains unclear. It has also rendered the information presented unverifiable directly, thereby hindering their further usage. This can be overcome only when checklists justify the inclusion and omission of each species by providing a valid reference/source for distribution records and the taxonomic treatise. The present communication is part of an effort to verify the validity of Indian reptile species listings, based on distributional records and a review of the earlier checklists of the Indian reptiles. In this paper, the species listings have been verified and species have been categorized based on the distributional records. A review of the checklists of Indian reptiles published over the past two decades has also been performed. Finally, a comprehensive list of lizards (Reptilia: Sauria) with valid distribution records in India has been provided along with source literature.

Recent developments (past 6 years) in the taxonomy and species occurrence information on Indian reptiles necessitates an update in the checklists of Indian lizards. Some of the new developments include records of Hemidactylus persicus from Gujarat (Vyas et al. 2006) and Japalura kaulbackii in Arunachal Pradesh (from Kunte \& Manthey 2009). Recent rediscoveries include that of Japalura sagittifera from Arunachal Pradesh (from Kunte \& Manthey 2009) and Lygosoma vosmaerii from Andhra Pradesh (Seetharamaraju et al. 2009). Doubts over the occurrence of $H$. karenorum in India (Zug et al. 2007; Mahony \& Zug 2008) and questions on the taxonomic validity of $H$. mahendrai and $H$. subtriedrus had been raised (Zug et al. 2007) and acknowledged by other workers (Giri \& Bauer 2008, Giri et al. 2009). The taxonomic revision of genus Mabuya (Mausfeld 2002), Cnemaspis anaikattiensis (Manamendra-Arachchi et al. 2007), C. kandiana (Wickramasinghe \& Munindradasa 2007), Phrynocephalus alticola (Barabanov \& Ananjeva 2007), Calotes andamanensis (Krishnan 2008), Teratolepis fasciatus (Bauer et al. 2008) and Dasia halianus (Wickramasinghe, submitted; Wickramasinghe, pers. comm.) from India have resulted in other changes. Description of new species included those of Cnemaspis australis, C. monticola and C. nilagirica (Manamendra- 
Arachchi et al. 2007), Hemidactylus aaronbaueri (Giri 2008), Hemidactylus sataraensis (Giri \& Bauer 2008), Calotes aurantolobium (Krishnan 2008), Hemidactylus treutleri (Mahony 2009a), Japalura otai (Mahony 2009b), and Hemidactylus gujaratensis (Giri et al. 2009). These have led to the revision of the list of lizards as provided by Das (2003).

\section{METHODS}

The list of lizards (Suborder Sauria) including the families Agamidae, Gekkonidae, Scincidae, Dibamidae, Anguidae, Eublepharidae, Lacertidae and Varanidae has been provided here. This list has been compiled primarily from articles published in scientific journals. I referred ca. 310 publications including technical reports, which formed the primary source for locality records. However reports in newsletters, unpublished reports, personal field observations and personal communications in writing (in lit.) with other herpetologists have also been taken into account. Information on species distribution and taxonomy has been complied from literature published until Sept 2009.

\section{Taxonomic Treatise}

The list provided, is at the species level and the taxonomy primarily follows Das (2003). Taxonomy of Hemidactylus albofasciatus follows Bauer et al. (2008) and the recognition of Mabuya as Eutropis follows Mausfeld et al. (2002). The species listed under the Genus Kaestlea (= Scincella) follows Eremchenko \& Das (2004). Validity of the listings has been reviewed for species as provided by the checklist of Indian reptiles (Murthy 1985; Murthy 1990a; Tikader \& Sharma 1992; Das 1997a; 2003), including its adjacent countries (Das 1994; Das 1996a; Sharma 2002). The words locality records and distributional records have been used synonymously.

\section{Validity of species listed}

Based on the distributional records available, the species have been classified into the following categories and a justification of the treatise has been provided through source literature and comments, wherever applicable.

(i) Species with valid distribution records within India. Distribution/Locality records within Indian limits currently available and have not been questioned by other workers.

(ii) Species reported from the regions politically disputed by India and Pakistan. This category includes species that are reported from politically disputed regions in Kashmir. The category has been created to acknowledge the current political situation in the areas from which we have valid distributional records.

(iii) Species whose distributional records are invalid or questioned. This category includes species for which distribution/locality records within India are available, but have been questioned. Also contains species whose inclusion has not been justified by providing source references/literature or relevant notes.

(iv) Species with unclear locality records. Species for which clear distribution/locality records within India are not available, but included in the checklists.

(v) Species known only from type specimen of unclear origin.

(vi) Species known only from type specimen, the original locality of which is not clear, but listed in earlier checklists.

(vii) Species valid in earlier lists, but omitted in this communication. Species that were earliervalid, butomitted in this communication due to the recent developments in taxonomic and distributional information.

The valid list of lizards in India, as represented by category A and B has separately been listed along with references corroborating their inclusion (Table 1). For the other categories, detailed comments have been provided along with a justification.

\section{Review of the checklists of Indian reptiles}

Validity of the species listed in the earlier checklists of India published in the past two decades has been reviewed. Based on existing and current information on their distribution, the following details have been reviewed for each of the publications -

(i) Erroneous inclusion of species without valid records and species whose distribution records were questioned.

(ii) Erroneous inclusion of species with unclear locality records.

(ii) Erroneous inclusion of species known only from type specimen of unknown origin.

(iv) Erroneous omission of valid species.

\section{RESULTS}

\section{Validity and categorization of lizard species based on distributional records}

The species that fall within the various categories as discussed in the methods section have been provided in the following paragraphs. The consolidated result, represented as the number of species classified under each category has been provided in Table 2 . The number of lizard species with valid distributional records from India, including those known from politically disputed regions (PDR) between India and Pakistan (Category $A$ and $B$ respectively), is currently 199 . These species have been listed in Table 1 along with a literature source corroborating each species' inclusion. However, in the past two decades, 17 (excluding category F) other species 
Table 1. List of Saurid reptilian species with valid distributional records within India (Category A).

\begin{tabular}{|c|c|c|}
\hline & Species & Source \\
\hline \multicolumn{3}{|c|}{ Agamidae } \\
\hline 1 & Bronchocela cristatella (Kuhl, 1820 ) & Smith $1935 a$ \\
\hline 2 & Bronchocela danieli (Tiwari \& Biswas, 1973) & Tiwari \& Biswas 1973 \\
\hline 3 & Bronchocela jubata Duméril \& Bibron, 1837 & Stoliczka 1873 \\
\hline 4 & Brachysaura minor (Hardwicke \& Gray, 1827) & Günther 1864 \\
\hline 5 & Bufoniceps laungwalansis (Sharma, 1978) & Sharma 1978 \\
\hline 6 & Calotes andamanensis Boulenger, 1891 & Krishnan 2008 \\
\hline 7 & Calotes aurantolabium Krishnan, 2008 & Krishnan 2008 \\
\hline 8 & Calotes calotes (Linnaeus, 1758) & Smith $1935 a$ \\
\hline 9 & Calotes ellioti Günther, 1864 & Günther 1864 \\
\hline 10 & Calotes emma Gray, 1845 & Pawar et al. 2004 \\
\hline 11 & Calotes grandisquamis Günther, 1875 & Günther 1875 \\
\hline 12 & Calotes jerdoni Günther, 1870 & Günther 1870 \\
\hline 13 & Calotes maria Gray, 1845 & Günther 1864 \\
\hline 14 & Calotes mystaceus Duméril \& Bibron, 1837 & Annandale 1904 \\
\hline 15 & Calotes nemoricola Jerdon, 1853 & Günther 1864 \\
\hline 16 & Calotes rouxii Duméril \& Bibron, 1837 & Günther 1864 \\
\hline 17 & Calotes versicolor (Daudin, 1802) & Günther 1864 \\
\hline 18 & Coryphophylax subcristatus (Blyth, 1860) & Günther 1864 \\
\hline 19 & Draco blanfordii Boulenger, 1885 & Biswas 1967 \\
\hline 20 & Draco dussumieri Duméril \& Bibron, 1837 & Günther 1864 \\
\hline 21 & Draco maculatus Gray, 1845 & Pawar 1999 \\
\hline 22 & Japalura andersoniana Annandale, 1905 & Smith 1935a \\
\hline 23 & Japalura kaulbackii Smith, 1937 & Kunte \& Manthey 2009 \\
\hline 24 & Japalura kumaonensis (Annandale, 1907) & Annandale 1907 \\
\hline 25 & Japalura major (Jerdon, 1870) & Annandale 1907 \\
\hline 26 & Japalura otai Mahony 2009 & Mahony 2009b \\
\hline 27 & Japalura planidorsata Jerdon, 1870 & Hora 1926 \\
\hline 28 & Japalura sagittifera Smith, 1940 & Kunte \& Manthey 2009 \\
\hline 29 & Japalura tricarinata (Blyth, 1854) & Hora 1926 \\
\hline 30 & Japalura variegata Gray, 1853 & Günther 1864 \\
\hline 31 & Laudakia agrorensis (Stoliczka, 1872) & Smith $1935 a$ \\
\hline 32 & Laudakia dayana (Stoliczka, 1871) & Smith 1935a \\
\hline 33 & Laudakia himalayana (Steindachner, 1867) & Schmidt 1926 \\
\hline 34 & Laudakia melanura Blyth, 1854 & Agrawal 1979 \\
\hline 35 & Laukadia tuberculata (Hardwicke \& Gray, 1827) & Günther 1864 \\
\hline 36 & Mictopholis austeniana (Annandale, 1908) & Annandale 1908 \\
\hline 37 & Otocryptis beddomii Boulenger, 1885 & Smith $1935 a$ \\
\hline 38 & Oriocalotes paulus Smith, 1935 & Smith $1935 a$ \\
\hline 39 & Phrynocephalus theobaldi Blyth, 1863 & Schmidt 1926 \\
\hline 40 & Psammophilus blanfordanus (Stoliczka, 1871) & Smith 1935a \\
\hline 41 & Psammophilus dorsalis (Gray in: Griffith \& Pidgeon, 1831) & Smith $1935 a$ \\
\hline 42 & Ptyctolaemus gularis (Peters, 1864) & Wall 1908a \\
\hline 43 & Salea anamallayana (Beddome, 1878) & Smith $1935 a$ \\
\hline 44 & Salea horsfieldii Gray, 1845 & Günther 1864 \\
\hline 45 & Sitana ponticeriana Cuvier, 1844 & Schmidt 1926 \\
\hline 46 & Trapelus agilis (Olivier, 1804) & Smith, 1935a \\
\hline 47 & Trapelus megalonyx Günther, 1864 & Prakash 1972 \\
\hline \multicolumn{3}{|c|}{ Anguidae } \\
\hline 48 & Ophisaurus gracilis (Gray, 1845) & Günther 1864 \\
\hline
\end{tabular}




\begin{tabular}{|c|c|c|}
\hline & Species & Source \\
\hline \multicolumn{3}{|c|}{ Chamaeleonidae } \\
\hline 49 & Chamaeleo zeylanicus Laurenti, 1768 & Günther 1864 \\
\hline \multicolumn{3}{|c|}{ Dibamidae } \\
\hline 50 & Dibamus nicobaricus (Fitzinger in Steindachner, 1867) & Tikader \& Das 1985 \\
\hline \multicolumn{3}{|c|}{ Eublepharidae } \\
\hline 51 & Eublepharis hardwickii Gray, 1827 & Smith $1935 a$ \\
\hline 52 & Eublepharis macularius (Blyth, 1854) & Smith $1935 a$ \\
\hline \multicolumn{3}{|c|}{ Gekkonidae } \\
\hline 53 & Calodactylodes aureus (Beddome, 1870) & Beddome 1870 \\
\hline 54 & Cnemaspis assamensis Das \& Sengupta, 2000 & Das \& Sengupta 2000 \\
\hline 55 & Cnemaspis australis Manamendra-Arachchi, Batuvita \& Pethiyagoda, 2007 & Manamendra-Arachchi et al. 2007 \\
\hline 56 & Cnemaspis beddomei (Theobald, 1876) & Beddome 1870 \\
\hline 57 & Cnemaspis goaensis Sharma, 1976 & Sharma 1976 \\
\hline 58 & Cnemaspis gracilis (Beddome, 1870) & Beddome 1870 \\
\hline 59 & Cnemaspis heteropholis Bauer, 2002 & Bauer 2002 \\
\hline 60 & Cnemaspis indica Gray, 1846 & Smith $1935 a$ \\
\hline 61 & Cnemaspis indraneildasii Bauer, 2002 & Bauer 2002 \\
\hline 62 & Cnemaspis jerdonii (Theobald, 1868) & Smith $1935 a$ \\
\hline 63 & Cnemaspis littoralis (Jerdon, 1854) & Beddome 1870 \\
\hline 64 & Cnemaspis mysoriensis (Jerdon, 1854) & Smith 1935a \\
\hline 65 & Cnemaspis monticola Manamendra-Arachchi, Batuvita \& Pethiyagoda, 2007 & Manamendra-Arachchi et al. 2007 \\
\hline 66 & Cnemaspis nairi Inger, Marx \& Koshy, 1984 & Inger et al. 1984 \\
\hline 67 & Cnemaspis nilagirica Manamendra-Arachchi, Batuvita \& Pethiyagoda, 2007 & Manamendra-Arachchi et al. 2007 \\
\hline 68 & Cnemaspis ornata (Beddome, 1870) & Beddome 1870 \\
\hline 69 & Cnemaspis otai Das \& Bauer, 2000 & Das \& Bauer 2000 \\
\hline 70 & Cnemaspis sisparensis (Theobald, 1876) & Smith $1935 a$ \\
\hline 71 & Cnemaspis tropidogaster (Boulenger, 1885) & Smith $1935 a$ \\
\hline 72 & Cnemaspis wynadensis (Beddome, 1870) & Beddome 1870 \\
\hline 73 & Cnemaspis yercaudensis Das \& Bauer, 2000 & Das \& Bauer 2000 \\
\hline 74 & Cosymbotus platyurus (Schneider, 1792) & Günther 1864 \\
\hline 75 & Crossobamon orientalis (Blanford, 1875) & Biswas \& Sanyal 1977a \\
\hline 76 & Cyrtodactylus adleri Das, 1997 & Das 1997b \\
\hline 77 & Cyrtodactylus fasciolatus (Blyth, 1860) & Smith 1935a \\
\hline 78 & Cyrtodactylus gubernatoris (Annandale, 1913) & Smith $1935 a$ \\
\hline 79 & Cyrtodactylus himalayanus Duda \& Sahi, 1978 & Duda \& Sahi 1978 \\
\hline 80 & Cyrtodactylus khasiensis (Jerdon, 1870) & Smith $1935 a$ \\
\hline 81 & Cyrtodactylus lawderanus (Stoliczka, 1871) & Smith $1935 a$ \\
\hline 82 & Cyrtodactylus malcomsmithi (Constable, 1949) & Murthy 1985 \\
\hline 83 & Cyrtodactylus mansarulus Duda \& Sahi, 1978 & Duda \& Sahi 1978 \\
\hline 84 & Cyrtodactylus rubidus (Blyth, 1861) & Stoliczka 1873 \\
\hline 85 & Cyrtodactylus stoliczkai (Steindachner, 1867) & Schmidt 1926 \\
\hline 86 & Cyrtopodion aravallensis (Gill, 1997) & Gill 1997 \\
\hline 87 & Cyrtopodion kachhense Stoliczka, 1872 & Smith $1935 a$ \\
\hline 88 & Cyrtopodion montiumsalsorum (Annandale, 1913) & Duda \& Sahi 1977 \\
\hline 89 & Cyrtopodion scabrum (von Heyden in Rüppell, 1827) & Smith 1935a \\
\hline 90 & Geckoella collegalensis (Beddome, 1870) & Beddome 1870 \\
\hline 91 & Geckoella deccanensis (Günther, 1864) & Günther 1864 \\
\hline 92 & Geckoella jeyporensis (Beddome, 1878) & Smith 1935a \\
\hline 93 & Geckoella nebulosa (Beddome, 1870) & Beddome 1870 \\
\hline 94 & Gehyra mutilata (Wiegmann, 1834) & Annandale 1904 \\
\hline 95 & Gekko gecko (Linnaeus, 1758) & Stoliczka 1873 \\
\hline
\end{tabular}




\begin{tabular}{|c|c|c|}
\hline & Species & Source \\
\hline 96 & Gekko smithi Gray, 1842 & Annandale 1904 \\
\hline 97 & Gekko verreauxi Tytler, 1865 (1864) & Smith $1935 a$ \\
\hline 98 & Hemidactylus aaronbaueri Giri, 2008 & Giri 2008 \\
\hline 99 & Hemidactylus albofasciatus Grandison \& Soman, 1963 & Grandison \& Soman 1963 \\
\hline 100 & Hemidactylus anamallensis (Günther, 1875) & Günther 1875 \\
\hline 101 & Hemidactylus bowringii (Gray, 1845) & Smith 1935a \\
\hline 102 & Hemidactylus brookii Gray, 1845 & Gleadow 1887 \\
\hline 103 & Hemidactylus flaviviridis Rüppell, 1835 & Smith 1935a \\
\hline 104 & Hemidactylus frenatus Duméril \& Bibron, 1836 & Stoliczka 1873 \\
\hline 105 & Hemidactylus garnotii Duméril \& Bibron, 1836 & Smith 1935a \\
\hline 106 & Hemidactylus giganteus Stoliczka, 1871 & Giri et al. 2003 \\
\hline 107 & Hemidactylus gracilis Blanford, 1870 & Smith $1935 a$ \\
\hline 108 & Hemidactylus gujaratensis Giri, Bauer, Vyas \& Patil 2009 & Giri et al. 2009 \\
\hline 109 & Hemidactylus leschenaultii Duméril \& Bibron, 1836 & Blanford 1871 \\
\hline 110 & Hemidactylus maculatus Duméril \& Bibron, 1836 & Günther 1864 \\
\hline 111 & Hemidactylus persicus Anderson, 1872 & Vyas et al. 2006 \\
\hline 112 & Hemidactylus porbandarensis Sharma, 1981 & Sharma 1981 \\
\hline 113 & Hemidactylus prashadi Smith, 1935 & Smith $1935 a$ \\
\hline 114 & Hemidactylus reticulatus Beddome, 1870 & Beddome 1870 \\
\hline 115 & Hemidactylus sataraensis Giri \& Bauer, 2008 & Giri \& Bauer 2008 \\
\hline 116 & Hemidactylus scabriceps (Annandale, 1906) & Smith $1935 a$ \\
\hline 117 & Hemidactylus triedrus (Daudin, 1802) & Smith 1935a \\
\hline 118 & Hemidactylus treutleri Mahony 2009 & Mahony 2009a \\
\hline 119 & Hemiphyllodactylus aurantiacus (Beddome, 1870) & Beddome 1870 \\
\hline 120 & Hemiphyllodactylus typus Bleeker, 1860 & Beddome 1870 \\
\hline 121 & Lepidodactylus lugubris (Duméril \& Bibron, 1836) & Stoliczka 1873 \\
\hline 122 & Phelsuma andamenense Blyth, 1861 (1860) & Stoliczka 1873 \\
\hline 123 & Ptychozoon kuhli Stejneger, 1902 & Günther 1864 \\
\hline 124 & Ptychozoon lionotum Annandale, 1905 & Pawar \& Biswas 2001 \\
\hline \multicolumn{3}{|c|}{ Lacertidae } \\
\hline 125 & Acanthodactylus cantoris Günther, 1864 & Günther 1864 \\
\hline 126 & Mesalina watsonana (Stoliczka, 1872) & Smith 1935a \\
\hline 127 & Ophisops beddomei (Jerdon, 1870) & Beddome 1870 \\
\hline 128 & Ophisops jerdoni Blyth, 1853 & Smith $1935 a$ \\
\hline 129 & Ophisops leschenaultii (Milne-Edwards, 1829) & Smith $1935 a$ \\
\hline 130 & Ophisops microlepis Blanford, 1870 & Smith $1935 a$ \\
\hline 131 & Ophisops minor Deraniyagala, 1971 & Smith 1935a \\
\hline 132 & Takydromus haughtonianus (Jerdon, 1870) & Smith $1935 a$ \\
\hline 133 & Takydromus khasiensis (Boulenger, 1917) & Smith $1935 a$ \\
\hline 134 & Takydromus sexlineatus Daudin, 1802 & Smith $1935 a$ \\
\hline \multicolumn{3}{|c|}{ Scincidae } \\
\hline 135 & Ablepharus grayanus (Stoliczka, 1872) & Smith $1935 a$ \\
\hline 136 & Ablepharus pannonicus (Fitzinger, 1823) & Sahi \& Duda 1986 \\
\hline 137 & Asymblepharus ladacensis (Günther, 1864) & Günther 1864 \\
\hline 138 & Asymblepharus himalayanum (Günther, 1864) & Günther 1864 \\
\hline 139 & Asymblepharus sikkimensis (Blyth, 1854) & Smith $1935 a$ \\
\hline 140 & Barkudia insularis Annandale, 1917 & Annandale 1917 \\
\hline 141 & Barkudia melanosticta (Schneider, 1801) & Ganapati \& Nayar 1952 \\
\hline 142 & Dasia nicobarensis Biswas \& Sanyal, 1977 & Biswas \& Sanyal 1977b \\
\hline 143 & Dasia olivacea Gray, 1839 & Stoliczka 1873 \\
\hline 144 & Dasia subcaeruleum (Boulenger, 1891) & Boulenger 1891 \\
\hline
\end{tabular}




\begin{tabular}{|c|c|c|}
\hline & Species & Source \\
\hline 145 & Eurylepis poonaensis (Sharma, 1970) & Sharma 1970 \\
\hline 146 & Eurylepis taeniolatus Blyth, 1854 & Smith 1935a \\
\hline 147 & Eutropis allapallensis (Schmidt, 1926) & Schmidt 1926 \\
\hline 148 & Eutropis andamanensis (Smith, 1935) & Smith 1935a \\
\hline 149 & Eutropis beddomei (Jerdon, 1870) & Smith 1935a \\
\hline 150 & Eutropis bibronii (Gray, 1838) & Smith 1935a \\
\hline 151 & Eutropis carinata (Schneider, 1801) & Smith 1935a \\
\hline 152 & Eutropis clivicola (Inger, Shaffer, Koshy \& Bakde, 1984) & Inger et al. 1984 \\
\hline 153 & Eutropis dissimillis (Hallowell, 1857) & Smith 1935a \\
\hline 154 & Eutropis gansi (Das, 1991) & Das 1991 \\
\hline 155 & Eutropis innotata (Blanford, 1870) & Smith 1935a \\
\hline 156 & Eutropis macularia (Blyth, 1853) & Smith 1935a \\
\hline 157 & Eutropis multifasciata (Kuhl, 1820) & Annandale 1904 \\
\hline 158 & Eutropis nagarjuni (Sharma, 1969) & Sharma 1971 \\
\hline 159 & Eutropis quadricarinata (Boulenger, 1887) & Murthy 1985 \\
\hline 160 & Eutropis rudis (Boulenger, 1887) & Biswas 1984 \\
\hline 161 & Eutropis rugifera (Stoliczka, 1870) & Stoliczka 1873 \\
\hline 162 & Eutropis trivittata (Hardwicke \& Gray, 1827) & Smith 1935a \\
\hline 163 & Eutropis tytlerii (Tytler in Theobald, 1868) & Stoliczka 1873 \\
\hline 164 & Lipinia macrotympanum (Stoliczka, 1873) & Stoliczka 1873 \\
\hline 165 & Lygosoma albopunctata (Gray, 1846) & Günther 1864 \\
\hline 166 & Lygosoma ashwamedhi (Sharma, 1969) & Sharma 1971 \\
\hline 167 & Lygosoma bowringii (Günther, 1864) & Smith 1935a \\
\hline 168 & Lygosoma goaensis (Sharma, 1976) & Sharma 1976 \\
\hline 169 & Lygosoma guentheri (Peters, 1879) & Smith 1935a \\
\hline 170 & Lygosoma lineata (Gray, 1839) & Smith 1935a \\
\hline 171 & Lygosoma pruthi (Sharma, 1977) & Sharma 1977 \\
\hline 172 & Lygosoma punctata (Gmelin, 1799) & Annandale 1915 \\
\hline 173 & Lygosoma vosmaerii (Gray, 1839) & Seetharamaraju et al. 2009 \\
\hline 174 & Novoeumeces schneiderii Daudin, 1802 & Vyas 1998 \\
\hline 175 & Ophiomorus raithmai Anderson \& Leviton, 1966 & Greer \& Wilson 2001 \\
\hline 176 & Ristella beddomii Boulenger, 1887 & Smith 1935a \\
\hline 177 & Ristella guentheri Boulenger, 1887 & Smith 1935a \\
\hline 178 & Ristella rurkii Gray, 1839 & Smith 1935a \\
\hline 179 & Ristella travancoricus (Beddome, 1870) & Beddome 1870 \\
\hline 180 & Kaestlea beddomei (Boulenger, 1887) & Smith 1935a \\
\hline 181 & Kaestlea bilineata (Gray, 1846) & Smith 1935a \\
\hline 182 & Kaestlea laterimaculata (Boulenger, 1887) & Smith 1935a \\
\hline 183 & Kaestlea palnica (Boettger, 1892) & Smith 1935a \\
\hline 184 & Kaestlea travancorica (Beddome, 1870) & Beddome 1870 \\
\hline 185 & Scincella macrotis (Fitzinger in: Steindachner, 1867) & Smith 1935a \\
\hline 186 & Sepsophis punctatus Beddome, 1870 & Beddome 1870 \\
\hline 187 & Sphenomorphus courcyanum (Annandale, 1912) & Smith 1935a \\
\hline 188 & Sphenomorphus dussumieri (Duméril \& Bibron, 1839) & Beddome 1870 \\
\hline 189 & Sphenomorphus indicus (Gray, 1853) & Wall 1908b \\
\hline 190 & Sphenomorphus maculatus (Blyth, 1853) & Annandale 1904 \\
\hline 191 & Tropidophorus assamensis Annandale, 1912 & Smith 1935a \\
\hline \multicolumn{3}{|c|}{ Uromastycidae } \\
\hline 192 & Uromastyx hardwickii Gray in Hardwicke \& Gray, 1827 & Günther 1864 \\
\hline \multicolumn{3}{|c|}{ Varanidae } \\
\hline 193 & Varanus bengalensis (Daudin, 1802) & Annandale 1915 \\
\hline
\end{tabular}




\begin{tabular}{|l|l|l|}
\hline & Species & Source \\
\hline 194 & Varanus flavescens (Hardwicke \& Gray, 1827) & Smith 1935a \\
\hline 195 & Varanus griseus Daudin, 1803 & Smith 1935b \\
\hline 196 & Varanus salvator Laurenti, 1768 & Annandale 1904 \\
\hline
\end{tabular}

Table 2. Family wise categorization of Indian lizard species based on distributional records

\begin{tabular}{|l|r|r|r|r|r|r|r|}
\hline Family / Category & \multicolumn{1}{c|}{ A } & \multicolumn{1}{c|}{ B } & \multicolumn{1}{c|}{ C } & \multicolumn{1}{c|}{ D } & E & F & Total \\
\hline Agamidae & 47 & 1 & 5 & 1 & & 2 & $\mathbf{5 6}$ \\
\hline Anguidae & 1 & & & & & & $\mathbf{1}$ \\
\hline Chamaeleonidae & 1 & & & & & & $\mathbf{1}$ \\
\hline Dibamidae & 1 & & & & & & $\mathbf{1}$ \\
\hline Eublepharidae & 2 & & & & & & $\mathbf{2}$ \\
\hline Gekkonidae & 71 & 1 & 4 & 1 & 1 & 7 & $\mathbf{8 5}$ \\
\hline Lacertidae & 10 & & 1 & & & & $\mathbf{1 1}$ \\
\hline Scincidae & 58 & 1 & 3 & & 1 & 3 & $\mathbf{6 6}$ \\
\hline Uromastycidae & 1 & & & & & & $\mathbf{1}$ \\
\hline Varanidae & 4 & & & & & & $\mathbf{4}$ \\
\hline Total & 196 & $\mathbf{3}$ & $\mathbf{1 3}$ & $\mathbf{2}$ & $\mathbf{2}$ & $\mathbf{1 2}$ & $\mathbf{2 2 8}$ \\
\hline
\end{tabular}

A - Species with valid distribution records within India B - Species reported from the regions politically disputed by India and Pakistan

C - Species whose distributional records are invalid or questioned

D - Species with unclear locality records

E - Species known only from type specimen of unclear origin

$\mathrm{F}$ - Species that were valid in earlier lists, but omitted in this

communication.

without any valid distributional records in India have been included in the various checklists of Indian reptiles. Details of these species falling under the other categories have been provided below, along with comments justifying the treatise.

\section{B. Species reported from the regions politically disputed by India and Pakistan}

Agamidae

Laudakia pakistanica Baig, 1989

Comments - Baig and Böhme (1996) described the subspecies Laudakia pakistanica khani from Chilas, an area that falls in a region politically disputed by India and Pakistan, in Kashmir. Das (1996a) commented that this species has been reported from politically disputed regions of India \& Pakistan.

\section{Gekkonidae}

Alsophylax boehmei Szczerbak, 1991

Comments - I have not seen the original species description. I follow the locality records (Skardu, Ladakh) provided by Khan (2002) and Das (1996a). Das (1996a) commented that this species has been reported from politically disputed regions of India \& Pakistan.

\section{Scincidae}

Asymblepharus tragbulense (Alcock, '1897' 1898)

Comments - I have not seen the original species description. The only known collection of the species was in 1885 from Tragbul Pass, about 50km NW Srinagar, presently in the politically disputed region between India and Pakistan (fide Das et al. 1998).
C. Species without valid records and species whose distribution records were questioned, but included in earlier checklist of Indian reptiles

Agamidae

Calotes bhutanensis Biswas, 1975

Comments - No known distribution records from India.

Tikader \& Sharma (1992) included this species for India.

Laudakia caucasia (Eichwald, 1831)

Comments - Locality records for this species (Kelat \& Bolan Pass) provided by Smith (1935a: 221) falls in Balochistan Province in Pakistan and there have been no other reported records within India subsequently. However this species has been included in the list of Indian reptiles provided by Das (1997a; 2003).

\section{Phrynocephalus luteoguttatus Boulenger, 1887}

Comments - No known locality records for India but included in the list of Indian reptiles by Das (1997a). I agree with Barabanov \& Ananjeva (2007) in not including India in the distributional range of this species.

\section{Phrynocephalus euptilopus Alcock \& Finn, '1896' 1897}

Comments - Das (1996b) has questioned the report of this species from deserts of Rajasthan (Daniel 1983; Tikader \& Sharma 1992; Daniel 2002; Sharma 2002). However, this species was included in earlier checklists (Murthy 1990; Tikader \& Sharma 1992; Das 1997a; Sharma 2002) of Indian reptiles. I agree with Bobrov (2005) and Barabanov \& Ananjeva (2007) in not including India in the distributional range of this species.

Salea kakhienensis (Anderson, '1878' 1879)

Comments - No known locality records for this species 
from India, but has erroneously been included for India in the lists by Das (1994; 1996a; 1997a).

\section{Gekkonidae}

Cyropodion fedtschenkoi (Strauch, 1987)

Comments - Das (1996b) questioned the reports of this species from deserts of Rajasthan (Tikader \& Sharma 1992; Sharma 1992). No known reports of this species from India.

\section{Cyrtopodion chitralense (Smith, 1935)}

Comments - Locality record for this species (Karakal) provided by Smith (1935a: 47) falls in North West Frontier Province of Pakistan and there have been no subsequent reports of this species from India. This species has been listed for India by Das (2003).

Cyrtopodion baturense (Khan \& Baig, 1992)

Comments - No valid records of this species from India but listed by Das (1997a).

\section{Teratoscincus microlepis Nikolski, 1899}

Comments - No known records of this species from India, but included for India by Murthy (1994).

\section{Lacertidae}

Acanthodactylus blanfordii Boulenger, 1918

Comments - No known locality records from India, but included for India by Das (1994; 1996a; 1997a).

\section{Scincidae}

Dasia grisea (Gray, 1845)

Comments - No known records of this species from India but included for India by Murthy (1985; 1994).

\section{Scincella reevesii (Gray, 1838)}

Comments - No known locality records from India, but included for India by Das (1994; 1996a; 1997a).

\section{Eutropis novemcarinata (Anderson, 1871)}

Comments - No known locality records from India, but included for India by Das (1997a; 2003).

\section{Species with unclear locality records listed in earlier checklists.}

Agamidae

Pseudocalotes microlepis (Boulenger, 1887)

Comments -Smith (1935a: 187) noted that the specimen recorded from Assam (Manipur?) by Annandale were lost. I have not verified if Annandale reported this species in his publications. However, Hallermann \& Bohme (2000) did not include India in the distributional range of this species. The presence of this species in India needs confirmation as there have been no other reports and the locality record for the specimen still remains uncertain. However, many lists of Indian reptiles have included this species.

Gekkonidae

Cyrtodactylus pulchellus Hardwicke \& Gray, 1827

Comments - No known locality records for this species from India. Das (2003) included this species in his list while denoting that it was not recorded from India specifically, but was cited by Smith (1935a: 38). It has been included in the lists by Das (1994; 1996a; 1997a; 2003). Given that the locality records are not available, the inclusion of this species needs confirmation.

E. Species known only from a type specimen, the original locality of which is not clear, but listed in earlier checklists.

Gekkonidae

Cnemaspis boei (Gray, 1842)

Comments - Known only from the type specimen, the locality record for which is not available (fide Smith 1935a: 75), and there have been no subsequent reports. However, Das (1994; 1996a; 1997a; 2003) included this species in the list of Indian reptiles. The presence of this species in India needs confirmation.

Scincidae

Eumeces blythianus (Anderson, 1871)

Comments - The type locality not known, but purchased in Amritsar (fide Smith 1935a: 340) and no subsequent reports, but was included in the lists provided by Das $(1994 ; 1997 a)$. The presence of this species in India needs confirmation.

F. Species valid in earlier lists, but omitted in this communication due to the recent developments in taxonomic and distributional information.

Agamidae

Phrynocephalus alticola Peters 1984

Comments - Following the taxonomy proposed by Barabanov \& Ananjeva (2007), this species has been treated as a subjective junior synonym of $P$. theobaldi, and not included in this list as a separate species.

\section{Phrynocephalus reticulatus (Eichwald, 1831)}

Comments - This species was erroneously reported to occur in Ladakh by Smith (1935a) and following this was included in subsequent checklists of India (Bobrov 2005).

\section{Gekkonidae}

Cnemaspis kandiana (Kelaart, 1852)

Comments - Based on taxonomy suggested by Wickremasinghe \& Munindradasa (2007), this species is confined to Sri Lanka and populations from India have been relegated to other species. 
Cnemaspis anaikattiensis Mukherjee, Bhupathy \& Nixon, 2005

Comments - Based on taxonomy suggested by Manamendra-Arachchi et al. (2007), this species is considered a subjective synonym of $C$. sisparensis.

Ptyodactylus homolepis Blanford, 1876

Comments - This species was erroneously reported by Sahi \& Duda (1985) to occur in Jammu \& Kashmir (Bobrov 2005).

Hemidactylus karenorum (Theobald, 1868)

Comments - Following Mahony et al. (2008) who raised doubts over the distribution of this species from India and awaiting confirmation of the only existing record from Cachar (Assam) Smith (1935a: 102), it has not been included in the list of Indian reptiles.

Hemidactylus mahendrai Shukla, 1983

Comments - Following the taxonomic changes suggested by Zug et al. (2007), this species has been treated as a synonym of $H$. brookii, and not included in the list as a separate species.

\section{Hemidactylus subtreidrus Jerdon, 1853}

Comments - The taxonomic validity of $H$. subtriedrus had been questioned by some (Zug et al. 2007) and acknowledged by other workers (Giri \& Bauer 2008; Giri et al. 2009). As a result, it has not been included in the list of Indian reptiles, awaiting taxonomic clarity.

\section{Teratolepis fasciata (Blyth, 1854 (1853))}

Comments-Based on recent taxonomic development, this species has been placed in Genus Hemidactylus and suggested a new name, $H$. imbricatus due to homonymy (Bauer et al. 2008). Also, it has been suggested by Bauer et al. (2008) that the reports from India are likely to be erroneous.

\section{Scincidae}

Chalcides pentadactylus Beddome, 1870

Comments - The type specimen reported from Beypur, Kerala is lost and its true status needs examination of fresh material (fide Smith, 1935a: 350). It has not been reported again since its original description and its presence in India needs confirmation.

\section{Dasia halianus (Haly and Nevill in: Nevill, 1887)}

Comments - The Indian Dasia halianus, has been taxonomically identified as Dasia subcaeruleum, while the distribution of Dasia halianus has been reported to be restricted to Sri Lanka (Wickramasinghe, submitted; Wickramasinghe, pers. comm.)

\section{Ophiomorus tridactylus (Blyth, 1853)}

Comments - There has been some confusion regarding the taxonomy and distribution, between this species and $O$. raithmai. However, only $O$. raithmai currently occurs in India, while the distribution of this species is limited to Afghanistan and Pakistan (Indraneil Das, Personal Communication). The locality records within India available for this species actually pertain to O. raithmai.

\section{Review of the checklists of Indian lizards}

In the checklists of Indian reptiles published over the past 20 years, the number of omissions of species has been greater than that of erroneous inclusions (Table 3). However, it must be noted that over the years there has been a decrease in the number of such omissions (But see Sharma 2002). Within erroneous inclusions, inclusion of species whose distribution records were invalid or were questioned has been high in lists provided by Das (1997a). The list of species erroneously included or omitted by published checklists is provided in Table 3 .

\section{DISCUSSION}

Brown (1992) made a plea for standardizing the distributional records of Indian reptiles almost two decades ago. However, drawing a standardized format for publishing species checklists is an important task to be undertaken, in order to verify and validate the species occurrence data and also to prevent perpetuation of mistakes. This is especially true for checklists of regional (different Indian states or protected area checklists for example) and national levels, which are primarily compilations. Annotated lists based on available locality records and justifying the inclusion or omission of species by providing relevant source literature or notes on specimens, could be a good way of validating regional and national level checklists. This would facilitate the possibility of verification of the information presented, thereby ensuring its quality and also duly pay credit to the deserving workers who generated the vouchered or otherwise substantiated records.

Quality of species occurrence data, as derived from species lists, significantly impacts conservation and management considerations. The Conservation Assessment and Management Plan (CAMP) for Indian reptiles (Molur \& Walker 1998) which formed the IUCN red list of Indian reptiles used the checklist list provided by Das (1997a) as the starting reference point for the number of reptiles in India. However, Das (1997a) contained many erroneous inclusions and omissions (See Table 3), and the standard of reporting does not provide means to directly verify the quality of information presented. Accurate and precise data on species occurrences are imperative for the assessment of conservation status and drawing management considerations. It is also pivotal for the species occurrence information to be accurate 
Table 3. Erroneous inclusion and omission of species in the checklists of Indian reptiles. The references have been arranged chronologically.

\begin{tabular}{|c|c|c|c|c|}
\hline Reference & $\begin{array}{l}\text { Species without } \\
\text { valid records / with } \\
\text { questionable records }\end{array}$ & $\begin{array}{l}\text { Species with } \\
\text { unclear locality } \\
\text { records }\end{array}$ & $\begin{array}{l}\text { Species } \\
\text { known only } \\
\text { from type } \\
\text { specimen } \\
\text { of unknown } \\
\text { origin }\end{array}$ & Omission of valid species \\
\hline Murthy 1985 & Dasia grisea & $\begin{array}{l}\text { Pseudocalotes } \\
\text { microlepis; }\end{array}$ & & $\begin{array}{l}\text { Bufoniceps laungwalansis; Laudakia melanura; } \\
\text { Trapelus megalonyx; Cnemaspis gracilis; C. nairi; C. } \\
\text { tropidogaster; Cyrtodactylus mansarulus; Cyrtopodion } \\
\text { montiumsalsorum; Gekko verreauxi; Hemidactylus } \\
\text { karenorum; H. porbandarensis; Lepidodactylus lugubris; } \\
\text { Ablepharus pannonicus; Chalcides pentadactlyus; } \\
\text { Lygosoma ashwamedhi; L. pruthi; Eutropis allapallensis; M. } \\
\text { clivicola; M. innotata; M. nagarjuni; M. rudis; Novoeumeces } \\
\text { schneiderii }\end{array}$ \\
\hline Murthy 1990 & $\begin{array}{l}\text { Phrynocephalus } \\
\text { euptilopus }\end{array}$ & $\begin{array}{l}\text { Pseudocalotes } \\
\text { microlepis }\end{array}$ & & $\begin{array}{l}\text { Trapelus megalonyx; Cnemaspis gracilis; C. nairi; } \\
\text { C.tropidogaster; Cyrtodactylus himalayanus; } \\
\text { C.malcomsmithii; C. mansarulus; Gekko verreauxi; } \\
\text { Lepidodactylus lugubris; Ablepharus pannonicus; } \\
\text { Chalcides pentadactlyus; Dasia haliana; Lygosoma } \\
\text { ashwamedhi; Eutropis allapallensis; M. clivicola; M. } \\
\text { innotata; M. nagarjuni; M. clivicola; Novoeumeces } \\
\text { schneiderii }\end{array}$ \\
\hline $\begin{array}{l}\text { Tikader \& } \\
\text { Sharma } 1992\end{array}$ & $\begin{array}{l}\text { Calotes bhutanensis; } \\
\text { Phrynocephalus } \\
\text { euptilopus; Cyrtopodion } \\
\text { fedtschenkoi }\end{array}$ & $\begin{array}{l}\text { Pseudocalotes } \\
\text { microlepis }\end{array}$ & $\begin{array}{l}\text { Lygosoma } \\
\text { vosmaerii }\end{array}$ & $\begin{array}{l}\text { Coryphophylax subcristatus; Laudakia melanura; Trapelus } \\
\text { megalonyx; Cnemaspis gracilis; C. nairi; C.tropidogaster; } \\
\text { Cyrtodactylus himalayanus; C.malcomsmithii; C. } \\
\text { mansarulus; Gekko verreauxi; Lepidodactylus lugubris; } \\
\text { Ablepharus pannonicus; Dasia haliana; Novoeumeces } \\
\text { schneiderii }\end{array}$ \\
\hline Murthy 1994 & $\begin{array}{l}\text { Salea kakhienensis; } \\
\text { Scincella reevesii; } \\
\text { Acanthodactylus } \\
\text { blanfordii }\end{array}$ & $\begin{array}{l}\text { Cyrtodactylus } \\
\text { pulchellus }\end{array}$ & $\begin{array}{l}\text { Cnemaspis } \\
\text { boei; } \\
\text { Eumeces } \\
\text { blythianus; } \\
\text { Lygosoma } \\
\text { vosmaerii }\end{array}$ & $\begin{array}{l}\text { Draco maculatus; Trapelus megalonyx; Cnemaspis gracilis; } \\
\text { Crossobamon orientalis }\end{array}$ \\
\hline Das 1994 & $\begin{array}{l}\text { Phrynocephalus } \\
\text { euptilopus; Teratoscincus } \\
\text { microlepis; Dasia grisea }\end{array}$ & & & $\begin{array}{l}\text { Laudakia melanura; Trapelus megalonyx; Cnemaspis } \\
\text { gracilis; C.tropidogaster; C. mansarulus; C. rubidus; Gekko } \\
\text { smithi; Hemidactylus porbandarensis; Lepidodactylus } \\
\text { lugubris; Ablepharus pannonicus; Chalcides pentadactlyus; } \\
\text { Eutropis allapallensis; M. clivicola; Novoeumeces } \\
\text { schneiderii }\end{array}$ \\
\hline Das 1996a & $\begin{array}{l}\text { Salea kakhienensis; } \\
\text { Scincella reevesii; } \\
\text { Acanthodactylus } \\
\text { blanfordii; }\end{array}$ & $\begin{array}{l}\text { Cyrtodactylus } \\
\text { pulchellus }\end{array}$ & $\begin{array}{l}\text { Cnemaspis } \\
\text { boei; } \\
\text { Lygosoma } \\
\text { vosmaerii }\end{array}$ & $\begin{array}{l}\text { Draco maculatus; Trapelus megalonyx; Cnemaspis } \\
\text { gracilis;Mabuya rugifera }\end{array}$ \\
\hline Das 1997a & $\begin{array}{l}\text { Salea kakhienensis; } \\
\text { Laudakia caucasia; } \\
\text { Phrynocephalus } \\
\text { luteoguttatus; } \\
\text { Phrynocephalus } \\
\text { euptilopus; Tenuidactylus } \\
\text { baturensis; Scincella } \\
\text { reevesii; Acanthodactylus } \\
\text { blanfordii; Mabuya } \\
\text { novemcarinata; }\end{array}$ & $\begin{array}{l}\text { Cyrtodactylus } \\
\text { pulchellus }\end{array}$ & $\begin{array}{l}\text { Cnemaspis } \\
\text { boei; } \\
\text { Eumeces } \\
\text { blythianus; } \\
\text { Lygosoma } \\
\text { vosmaerii }\end{array}$ & $\begin{array}{l}\text { Draco maculatus; Trapelus megalonyx; Crossobamon } \\
\text { orientalis; Cyrtopodion montiumsalsorum; Eurylepis } \\
\text { poonaensis }\end{array}$ \\
\hline Sharma 2002 & $\begin{array}{l}\text { Calotes bhutanensis; } \\
\text { Phrynocephalus } \\
\text { euptilopus; Cyrtopodion } \\
\text { fedtschenkoi }\end{array}$ & & & $\begin{array}{l}\text { Laudakia melanura; Trapelus megalonyx; Cyrtodactylus } \\
\text { himalayanus; C.malcomsmithii; C. mansarulus; Gekko } \\
\text { verreauxi; Hemiphyllodactylus typhus, Ptychozoon } \\
\text { lionotum; Ablepharus pannonicus; Barkudia melanosticta; } \\
\text { Dasia haliana; Novoeumeces schneiderii }\end{array}$ \\
\hline Das 2003 & $\begin{array}{l}\text { Laudakia caucasia; } \\
\text { Cyrtodactylus chitralensis; } \\
\text { Mabuya novemcarinata }\end{array}$ & $\begin{array}{l}\text { Cyrtodactylus } \\
\text { pulchellus }\end{array}$ & $\begin{array}{l}\text { Cnemaspis } \\
\text { boei }\end{array}$ & Draco maculatus \\
\hline
\end{tabular}


for it to be used for further research purposes such as predictions on species distributions, habitat suitability, and threat assessments etc. While there have been global efforts to share and provide free access to species distribution information (Ex. GBIF 2008), the current reporting standards of publications on Indian reptiles (regional and national) actually hinder further usage of the information presented. This could be ameliorated only if individual workers and publishing houses/journals present annotated checklists that contain source literature and details substantiating the inclusion and omission of each species.

\section{REFERENCES}

Agrawal, H.P. (1979). A checklist of reptiles of Himachal Pradesh, India. The Indian Journal of Zootomy 20(2): 115-124.

Annandale, N. (1904). Contributions to Oriental Herpetology I - The lizards of the Andamans, with the description of a new gecko and a note on the reproduced tail in Ptychozoon homalocephalum. Journal of Asiatic Society of Bengal 73(2) (Suppl): 13-22.

Annandale, N. (1907). Reports on a collection of batrachia, reptiles and fish from Nepal and the western Himalayas Himalayan Lizards. Records of the Indian Museum 1(10): 151-155.

Annandale, N. (1908). Description of a new species of lizard of the genus Salea from Assam. Records of the Indian Museum 2: $37-38$.

Annandale, N (1915). Fauna of the Chilka Lake - Mammals, Reptiles and Batrachians. Memoirs of the Indian Museum 5: 165-173.

Annandale, N. (1917). A new genus of limbless skinks from an Island in the Chilka Lake. Records of the Indian Museum 8: 17-21.

Baig, K.J. \& W. Böhme (1996). Description of two new subspecies of Laudakia pakistanica (Sauria: Agamidae). Russian Journal of Herpetology 3(1): 1-10.

Barabanov, A.V. \& N.B. Ananjeva (2007). Catalogue of the available scientific species-group names for lizards of the genus Phrynocephalus Kaup, 1825 (Reptilia, Sauria, Agamidae). Zootaxa 1399: 1-56.

Bauer, A.M (2002). Two new species of Cnemaspis (Reptilia: Squamata: Geckonidae) from Gund Uttar Kannada India. Mitteilungen Hamburg Zoological Museum \& Institution 99: 155-167.

Bauer, A.M., V.B. Giri, E. Greenbaum, T.R. Jackman, M.S. Dharne, \& Y.S. Shouche (2008). On the systematics of the gekkonid genus Teratolepis Günther, 1869: another one bites the dust. Hamadryad 32: 13-27.

Beddome, R.H. (1870). Descriptions of some new lizards from the Madras Presidency. Madras Monthly Journal of Medical Science 1: 30-35.

Biswas, S. (1967). Occurrence of Draco blanfordi Boulenger (Sauria: Agamidae) in Assam India. Journal of Bombay Natural History Society 64: 574.

Biswas, S. (1984). Some notes on the reptiles of Andaman \& Nicobar Islands. Journal of Bombay Natural History Society 81: 476-481.

Biswas, L. N. \& D.P. Sanyal (1977a). Fauna of Rajasthan, India, part: Reptilia. Records of Zoological Survey of India 73: 247269.

Biswas, S. \& D.P. Sanyal (1977b). A new species of skink of the genus Dasia Gray 1889 [Reptilia: Scincidae] from Car
Nicobar Islands India. Journal of Bombay Natural History Society 74(1): 133-136.

Blanford, W.I. (1871). Note on Hemidactylus marmoratus $H$. keelarti Theobald and Ablabes humerti. Proceedings of the Asiatic Society 5: 173-174.

Bobrov V.V. (2005). Independence of the central Asian faunistic region (according to the distribution of lizards (Reptilia Sauria)). Biology Bulletin 32 (6): 276-289. Translated from Izvestiya Academeii Nauk Seriya Biologicheskaya 6: 694 709.

Boulenger G.A. (1891). Description of a new species of lizard obtained by Mr. H. S. Ferguson in Travancore South India. Journal of Bombay Natural History Society 6(4): 449.

Brown, S.B. (1992). Indian herpetofauna: a plea for standardized distributional records. Hamadryad 17: 52-53.

Chapman, A.D. (2005a). Uses of Primary Species-Occurrence Data version 1.0. Report for the Global Biodiversity Information Facility, Copenhagen. Available at http://www2. gbif.org/UsesPrimaryData.pdf. Accessed on $10^{\text {th }}$ July 2008.

Chapman, A.D. (2005b). Principles of Data Quality version 1.0. Report for the Global Biodiversity Information Facility, Copenhagen. Available at http://www2.gbif.org/DataQuality. pdf. Accessed on $10^{\text {th }}$ July 2008.

Daniel, J.C. (1983). Book of Indian Reptiles and Amphibians. Oxford University Press, Oxford, 235pp.

Daniel, J C. (2002). The Book of Indian Reptiles. Bombay Natural History Society, Bombay, 141pp.

Das, I. (1991). A new Species of Mabuya from Tamil Nadu State southern India (Squamata:Scincidae). Journal of Herpetology 25(3): 342-344.

Das, I. (1994). The reptiles of South Asia: Checklist and distributional summary. Hamadryad 19: 15-40.

Das, I. (1996a). Biogeography of the Reptiles of south Asia. Krieger Publishing Company, Florida, $87 p p+36$ plates.

Das, I. (1996b). Handbook: Indian lizards [Book Review]. Herpetological Review 27: 44-46.

Das, I. (1997a). Checklist of the reptiles of India with English common names. Hamadryad 22(1): 32-45.

Das, I. (1997b). A new species of Cyrtodactylus from the Nicobar Islands India. Journal of Herpetology 31(3): 375-382.

Das, I. (2003). Growth of knowledge on the reptiles of India with an introduction to systematics taxonomy and nomenclature. Journal of Bombay Natural History Society 100(2\&3): 446501.

Das, I. \& A.M. Bauer (2000). Two new species of Cnemaspis (Sauria: Gekkonidae) from southern India. Russian Journal of Herpetology $7(1)$ : 17-28.

Das, I. \& S. Sengupta (2000). A new species of Cnemaspis (Sauria: Gekkonidae) from Assam north-eastern India. Journal of South Asian Natural History 5(1): 17-24.

Das, I., B, Dattagupta \& N.C. Gayen (1998). Systematic status of Lygosoma himalayanum tragbulensis Alcock "1897" 1898 (Sauria: Scincidae) collected by the Pamir Boundary Commission 1885. Russian Journal of Herpetology 5(2): 147-150

Duda, P.L. \& D.N. Sahi (1977). An uptodate checklist of herpetiles of Jammu \& Kashmir. Jammu and Kashmir University Review 6(10): 1-7.

Duda, P.L. \& D.N. Sahi (1978). Cyrtodactylus himalayanus: A new gekkonid species from Jammu India. Journal of Herpetology 12(3): 351-354.

Eremchenko, V.M. \& I. Das (2004). Kaestlea: A new genus of scincid lizards (Scincidae: Lygosominae) from the Western Ghats south-western India. Hamadryad 28(1\&2): 43-50.

Ganapati, P. N. \& K.K. Nayar (1952). Occurrence of the limbless lizard Barkudia Annandale at Waltair. Current Science 21: 105-106.

Gayen, N.C. (1999). A synopsis of the reptiles of Gujarat, western India. Hamadrayad 24: 1-22. 
Gill, E.V.S (1997). Cyrtodactylus aravallensis a new Gekkonidae from the Delhi ridge. Journal of Bombay Natural History Society 94(1): 122-123 + II plates.

Giri, V.B. (2008). A new rock dwelling Hemidactylus (Squamata: Gekkonidae) from Maharashtra India. Hamadryad 32: 2533.

Giri, V.B. \& M.A. Bauer (2008). A new ground-dwelling Hemidactylus (Squamata: Gekkonidae) from Maharashtra with a key to the Hemidactylus of India. Zootaxa 1700: 2134.

Giri, V., A.M. Bauer \& N. Chaturvedi (2003). Notes on the distribution, natural history and variation of Hemidactylus giganteus Stoliczka, 1871. Hamadryad 27(2): 217-221.

Gleadow, F. (1887). Description of a new lizard from the "Dangs." Journal of Bombay Natural History Society 2: 49-51.

Giri, V.B., A.M. Bauer, R. Vyas \& S. Patil (2009). New Species of Rock-Dwelling Hemidactylus (Squamata: Gekkonidae) from Gujarat, India. Journal of Herpetology 43(3): 385-393.

GBIF (2008). Global Biodiversity Information Facility. www.gbif org. Accessed on July 2008.

Grandison A.G.C. \& P.W. Soman (1963). Description of a new geckonid lizard from Maharashtra India. Journal of Bombay Natural History Society 60(2): 322-325 + II plates.

Greer E.A. \& G.D.F. Wilson (2001). Comments on the scincid lizard genus Ophiomorus with a cladistic analysis of the species. Hamadryad 26(2): 261-271.

Günther A.C.L.G. (1864). The Reptiles of British India. R Hardwicke, London, 444pp+26 plates.

Günther A.C.L.G. (1870). Description of a new Indian lizard of the genus Calotes. Proceedings of the Zoological Society of London $778-779+1$ plate.

Günther A.C.L.G. (1875). Second report on collection of Indian Reptiles obtained by the British Museum. Proceedings of the Zoological Society of London 224-234+4 plates.

Hallermann, J. \& W. Böhme (2000). A review of the genus Pseudocalotes (Squamata: Agamidae), with description of a new species from West Malaysia. Amphibia-Reptilia 21: 193-210.

Hora S. L (1926). Part II - On the unnamed collection of lizards of the family Agamidae. Records of the Indian Museum 28: 215-220.

Inger R.F., H.B. Shaffer, M. Koshy \& R. Badke (1984). A report on the collection of Ambhibians and reptiles from the Ponmudi Kerala South India. Journal of Bombay Natural History Society 81(3): 551-570.

Khan, M.S (2002). Key and checklist to the lizards of Pakistan. Herpetozoa 15: 179-199.

Krishnan, S (2008). New Species of Calotes (Reptilia: Squamata: Agamidae) from the Southern Western Ghats India. Journal of Herpetology 42(3): 530-535.

Kunte, K. \& U. Manthey (2009). Rediscovery of Japalura sagittifera (Sauria: Agamidae) from the Eastern Himalayas, Arunachal Pradesh: An addition to the Indian Herpetofauna. Sauria 31: 49-55.

Mahony, S (2009a). A new species of Gecko of the Genus Hemidactylus (Reptilia: Gekkonidae) from Andhra Pradesh. Russian Journal of Herpetology 16(1): 27-34.

Mahony, S(2009b). Anew species of Japalura(Reptilia:Agamidae) from northeast India with a discussion of the similar species Japalura sagittifera Smith, 1940 and Japalura planidorsata Jerdon, 1870. Zootaxa 2212: 41-61.

Mahony, S. \& G.R. Zug (2008). Hemidactylus karenorum (Squamata, Gekkonidae) in India. Hamadryad 32: 84-86.

Manamendra-Arachchi, K., S. Batuwita \& R. Pethiyagoda (2007). A taxonomic revision of the Sri Lankan day-geckos (Reptilia: Gekkonidae: Cnemaspis), with description of new species from Sri Lanka and southern India. Zeylanica 7 (1) 9-122

Mausfeld, P., A. Schmitz, W. Böhme, B. Misof, D. Vrcibradic
\& C.F.D. Rocha (2002). Phylogenetic Affinities of Mabuya atlantica Schmidt, 1945, Endemic to the Atlantic Ocean Archipelago of Fernando de Noronha (Brazil): Necessity of Partitioning the Genus Mabuya Fitzinger, 1826 (Scincidae: Lygosominae). Zoologischer Anzeiger 241: 281-293.

Molur, S. \& S. Walker (1998) (eds). Report of BCCP CAMP on Reptiles of India. Zoo Outreach Organisation, Coimbatore, India, 65p.

Murthy, T.S.N. (1985). Classification and distribution of the reptiles of India. The Snake 17: 48-71.

Murthy, T.S.N. (1990). A field book of the lizards of India. Records of the Zoological Survey of India. Occasional paper 115: 1-116.

Murthy, T.S.N. (1994). An updated handlist of the reptiles of India. Cobra 17: 17-37.

Pawar, S.S. (1998). Biogeography of the reptiles of South Asia [Book review]. Current Science 75(8): 857-858.

Pawar, S.S. (1999). Effect of habitat alteration on the herpetofaunal assemblages of evergreen forests in Mizoram North-east India. Unpublished Masters Dissertation submitted to Saurashtra University Rajkot, India, 56pp.

Pawar, S.S. \& S. Biswas (2001). First record of the Smoothbacked Parachute Gecko Ptychozoon lionotum Annandale 1905 from the Indian Mainland. Asiatic Herpetological Research 9: 101-106

Pawar, S.S., G.S. Rawat \& B.C. Choudhury (2004). Recovery of frog and lizard communities following primary habitat alteration in Mizoram Northeast India. BMC Ecology 4(1): 10.

Prakash, I. (1972). Notes on little known lizards from the Rajasthan desert. Journal of Bombay Natural History Society 69(2): 424-428.

Sahi, D.N. \& P.L. Duda (1985). A checklist and keys to the amphibians and reptiles of Jammu and Kashmir State, India. Bulletion of Chicago Herpetological Society 20(3\&4): 86-97.

Sahi D.N. \& P.L. Duda (1986). Occurrence of Ablepharus pannonicus Fitzinger (Lacertilia: Scincidae) in Jammu: An addition to the Indian reptilian fauna. Bulletin of Chicago Herpetological Society 21(3\&4): 92-93.

Schmidt K.P (1926). Amphibians and reptiles of the James Simpson-Roosevelt Asiatic expedition. Fieldana Zoology 12(13): 167-173

Seetharamaraju, M., R. Sreekar, C. Srinivasulu, B. Srinivasulu, H. Kaur \& P. Venkateshwarlu (2009). Rediscovery of Vosmer's Writhing Skink Lygosoma vosmaerii (Gray, 1839) (Reptilia: Scincidae) with a note on its taxonomy. Journal of Threatened Taxa 1(12): 624-626.

Sharma, R.C. (1970). A new lizard Eumeces poonaensis (Scincidae) from India. Records of the Zoological Survey of India 63(3\&4): 239-241.

Sharma, R.C. (1971). The reptilian fauna of the Nagarjuna Dam area (Andhra Pradesh India). Records of the Zoological Survey of India 63: 77-93.

Sharma, R.C. (1976). Records of the reptiles of Goa. Records of the Zoological Survey of India 71: 151-167.

Sharma R.C. (1977). A new lizard of the genus Riopa Gray (Scincidae) from Tamil Nadu, India. Records of the Zoological Survey of India 73(1-4): 41-44.

Sharma, R.C. (1978). A new species of Phrynocephalus Kaup (Reptilia: Agamidae) from the Rajasthan desert India with notes on its ecology. Bulletin of the Zoological Survey of India 1(3): 291-294.

Sharma, R.C. (1981). Hemidactylus porbandarensis a new geckonid lizard from Gujarat India. Bulletin of the Zoological Survey of India 4(1): 1-2.

Sharma R.C. (2002). The fauna of India and the adjacent countries - Reptilia (Sauria). Vol II. Zoological Survey of India, Kolkatta, 430pp.

Smith M.A. (1931). The Fauna of British India including Ceylon 
and Burma. Vol. I. Loricata Testudines. Taylor and Francis, London, $185 \mathrm{pp}+2$ plates.

Smith M.A. (1935a). The Fauna of British India including Ceylon and Burma. Reptilia and Amphibia. Vol. II Sauria. Taylor and Francis, London, $440 p p+1$ plate.

Smith, M.A. (1935b). Some notes on the monitors. Journal of Bombay Natural History Society 35: 615-619.

Smith, M.A. (1943). The Fauna of British India Ceylon and Burma including the whole of the Indo-Chinese region. Vol. III. Serpentes. Taylor and Francis, London, 583pp+1 map.

Stoliczka, F. (1873). Notes on some Andamanese and Nicobarese reptiles with the descriptions of three new species of lizards. Journal of the Asiatic Society of Bengal 42(3): 162-169.

Tikader, B.K. \& A.K. Das (1985). Glimpses of animal life in the Andaman and Nicobar Islands. Zoological Society of India, Kolkatta, 170pp.

Tikader B.K. \& R.C. Sharma (1992). Handbook of Indian Reptiles. Zoological Survey of India, Kolkatta, 250pp+42 plates.

Tiwari, K. \& S. Biswas (1973). Two new reptiles from the Great Nicobar Island. Journal of Zoological Society of India 25(1\&2): 57-63.

Vyas, R. (1998). The reptiles of Gujarat state: Updated distribution. Tiger paper 25(1): 8-14.
Vyas, R. (2000). Comments on 'A synopsis of the reptiles of Gujarat India'. Hamadryad 25(2): 203-207.

Vyas, R., V. Giri \& A.M. Bauer (2006). First record of Hemidactylus persicus Anderson 1872 (Squamata: Sauria: Gekkonidae) from the Republic of India with notes on its distribution. Hamadryad 30(1\&2): 209-211.

Wall, F. (1908a). Remarks on Agamid lizard (Ptyctolaemus gularis). Journal of Bombay Natural History Society 18: 505.

Wall F. (1908b). Viviparous habit of the common Indian skink (Lygosoma indica). Journal of Bombay Natural History Society 18: 505

Wickramasinghe, L.J.M. \& D.A.I. Munindradasa (2007). Review of the genus Cnemaspis Strauch, 1887 (Sauria: Gekkonidae) in Sri Lanka with the description of five new species. Zootaxa 1490: 1-63.

Wickramasinghe, L.J.M (submitted). A comparative study of Dasia halianus in India and Sri Lanka. Journal of Threatened Taxa.

Zug, G.R., Vindum, J.V. \& M.S. Koo (2007). Burmese Hemidactylus (Reptilia Squamata Gekkonidae): taxonomic notes on tropical Asian Hemidactylus. Proceedings of the California Academy of Sciences 58: 387-405. 